\title{
CARACTERÍSTICAS CLÍNICAS DEL DOLOR EN ADULTOS MAYORES DE LA FUNDACIÓN SANTA SOFÍA EN BOGOTÁ D.C.
}

\section{CLINICAL FEATURES OF PAIN IN THE FUNDATION SANTA SOFIA'S ELDER PEOPLE AT BOGOTÁ D.C.}

\begin{abstract}
Juan Carlos Morales Ruiz', Edgar Orlando Cote Rojas²
${ }^{1}$ Médico Cirujano. Especialista en Gerontología Social. Magíster en Educación. Investigador Grupo Envejecimiento y Vejez Facultad de Medicina U.D.C.A. Decano Facultad de Medicina Universidad de Ciencias Aplicadas y Ambientales U.D.C.A. Dirección: Calle 222 No 55 - 37. Correo Electrónico: ju.morales@udca.edu.co ² Médico Cirujano. Especialista en Gerontología Social. Candidato a Maestría en Educación Investigador U.D.C.A. Fundación Universitaria del Área Andina.
\end{abstract}

Rev. U.D.C.A Act. \& Div. Cient. 13 (2) 7-14, 2010

\section{RESUMEN}

El dolor es una de las quejas más comunes en la consulta médica de atención primaria. La frecuencia del síntoma en la población geriátrica hace que sea considerado, en la actualidad, como un problema sanitario de proporciones epidémicas, que afecta la calidad de vida del individuo y representa grandes pérdidas económicas, derivadas del costo de la atención médica y de la disminución de la funcionalidad; no obstante, existen insuficientes estudios orientados a describir el fenómeno y establecer parámetros para el diagnóstico y el tratamiento del dolor, en este grupo etáreo. Este trabajo aporta información acerca de las características del dolor y de las estrategias utilizadas para el abordaje del síntoma, en un grupo de más de mil ancianos no institucionalizados, de la ciudad de Bogotá, mediante la revisión de registros clínicos, obtenidos en el período comprendido entre enero de 2001 y diciembre de 2007. Los resultados logrados coinciden con los referentes existentes en la literatura, registrándose una preocupante falta de atención, por los aspectos sicológico y social del cuadro.

Palabras clave: Dolor, anciano, aspectos clínicos, diagnóstico, terapéutica.

\section{SUMMARY}

Pain is one of the most common complaints in the primary care setting. The frequency in the geriatric population is such, that it is considered a sanitary problem of epidemic proportions, which affects the quality of life of the individual and represents a big economic burden, related with the cost of the medical attention and the decrease of the functionality. At the present there are only a few studies which describe the phenomenon and establish parameters for the diagnosis and the treatment of the pain in the elderly. This study provides some information about the characteristics of the pain and the strategies used for the diagnosis and the treatment of the symptom in a group of more than one thousand not institutionalized persons at Bogota City, in the period between January, 2001 and December, 2007. The information obtained trough the clinical registries coincides with those registered in the literature, observing a worrying absence of attention of the psychological and social aspects of the scene.

Key words: Pain, elderly people, clinical issues, diagnosis, therapy.

\section{INTRODUCCIÓN}

El dolor es un síntoma frecuente en los pacientes geriátricos (Gregorio Gil et al. 2007). Más de tres cuartas partes de los mayores de setenta y cinco años manifiesta haber experimentado algún tipo de dolor y cerca de dos terceras partes de este mismo grupo, residentes en hogares de ancianos, se ven afectados por dolor crónico (Clark \& Cox, 2002).

La evaluación del paciente con dolor es una tarea compleja, dada la subjetividad de la experiencia nociceptiva y la dificultad para establecer un código único de comunicación, que permita la interpretación clara de un fenómeno de naturaleza individual. En tal sentido, diferentes autores plantean que la valoración del dolor debe incluir las dimensiones sensorial, 
afectiva y cognitiva del paciente (Catananti \& Gambassi, 2010; Gregorio Gil et al. 2007; Katz, 1999).

De acuerdo con las últimas guías de la Sociedad Americana de Anestesiología para el manejo del dolor crónico, el abordaje integral de estos pacientes debe estar fundamentado en cuatro pilares: la anamnesis, el examen clínico, la valoración psicosocial y las pruebas diagnósticas complementarias (Am. Soc. Anesthesiol. Task Force on Chronic Pain Management, 2010).

Entre los métodos utilizados para mejorar la comprensión del fenómeno doloroso, optimizar el diagnóstico y garantizar el seguimiento adecuado de la evolución del paciente, se encuentran las escalas de medición y de caracterización del dolor, el cuestionario de manejo del dolor, el inventario de manejo del dolor crónico, el cuestionario computarizado del dolor y el cuestionario del dolor de McGill, de acuerdo con los referentes que incluyen (Dijkers, 2010; Borsook et al. 1999). Los mismos autores mencionan que las más utilizadas en la práctica clínica, dada la facilidad de su aplicación e interpretación, están orientadas a valorar únicamente la intensidad del síntoma. Las segundas, usadas con mayor frecuencia en estudios de investigación y en centros de manejo especializado del dolor, garantizan una comprensión multidimensional de la experiencia dolorosa, pero requieren una mayor disponibilidad de tiempo para la aplicación y un entrenamiento especializado para la interpretación.

La caracterización de los mecanismos que subyacen a la aparición del síntoma, ha permitido mejorar la comprensión del proceso nociceptivo y la calidad de las intervenciones terapéuticas. Una estrategia óptima de manejo debe incluir medidas tendientes a equilibrar las alteraciones orgánicas y abordar los fenómenos sicológicos y sociales, relacionados con el mismo (Am. Soc. Anesthesiol. Task Force on Chronic Pain Management, 2010; Gottschalk E Smith, 2001).

Los principios básicos para el manejo del dolor incluyen la valoración de todas las alternativas terapéuticas existentes, el inicio temprano del tratamiento, el seguimiento de la evolución, los ajustes requeridos en el desarrollo del cuadro, la combinación de métodos farmacológicos y no farmacológicos, la selección de un esquema individual de tratamiento y el mantenimiento de la terapia hasta alcanzar la resolución definitiva del cuadro de base (Am. Soc. Anesthesiol. Task Force on Chronic Pain Management, 2010; Gottschalk E Smith, 2001; Hadjistavropoulus et al. 2007).

Las acciones no farmacológicas del dolor comprende la implementación de medidas de autocuidado y la aplicación de técnicas y de procedimientos enfocados a minimizar el impacto biológico, sicológico y social del cuadro. Entre las actividades que han demostrado ser útiles en el procedimiento del paciente con dolor, se encuentran la promoción del auto cuidado, la sensibilización del paciente y su familia en relación con las características de la enfermedad y con la utilización de procesos que garanticen la recuperación funcional la independencia del paciente (Am. Soc. Anesthesiol. Task Force on Chronic Pain Management, 2010; Marcus, 2000; Turk E Okifuji, 2001).

La tendencia actual en la práctica farmacológica del dolor está orientada hacia el control de los factores orgánicos y sicológicos, involucrados en la génesis del cuadro. Los medicamentos aplicados con mayor frecuencia en la práctica cotidiana son los analgésicos no opiáceos, los analgésicos opiáceos y los agentes coadyuvantes (Am. Soc. Anesthesiol. Task Force on Chronic Pain Management, 2010; Gregorio Gil et al. 2007; Christo, 2003; Ashburn E Staats, 1999).

Entre los primeros, se destacan los antiinflamatorios no esteroideos (AINEs), agentes farmacológicos indicados en el manejo del dolor agudo y crónico no maligno, de leve a moderada intensidad, que constituyen la primera opción, en forma aislada o en combinación, de la escalera terapéutica de la Organización Mundial de la Salud, en el manejo del dolor asociado con cáncer (Am. Soc. Anesthesiol. Task Force on Chronic Pain Management, 2010; Loza, 2008; Christo, 2003).

Una de las principales limitaciones del uso de los AINEs está relacionada con la aparición frecuente de efectos adversos, especialmente, en los casos de dolor crónico (Loza, 2008). El acetaminofén constituye la terapia de elección en los pacientes que presentan contraindicaciones (hipersensibilidad, patologías previas o idiosincrasia) y/o intolerancia a los AINEs (Christo, 2003; Herr \& Garand, 2001).

El uso de opiáceos, se encuentra previsto en el segundo y en el tercer paso de la escalera analgésica, para el tratamiento del dolor agudo severo y del dolor crónico moderado a severo (Am. Soc. Anesthesiol. Task Force on Chronic Pain Management, 2010). El efecto de estos fármacos esta por el bloqueo de receptores opioides (mu, delta y kappa), que reducen la intensidad de las señales dolorosas en el sistema nervioso periférico y central (Christo, 2003; Herr \& Garand, 2001).

En aquellos pacientes que no responden en forma adecuada a la terapia farmacológica, se recomienda el uso de técnicas invasivas de control del dolor, entre las cuales, se reconocen los bloqueos nerviosos, la infiltración local con anestésicos o esteroides, la administración epidural y/o intratecal de opiáceos y la estimulación neural, mediante la implantación de electrodos en áreas adyacentes a los nervios periféricos o a la médula espinal (Am. Soc. Anesthesiol. Task Force on Chronic Pain Management, 2010; Ashburn E Staats, 1999). 


\section{MATERIALES Y MÉTODOS}

El estudio, de tipo descriptivo y de carácter exploratorio, se basó en la revisión de los registros clínicos de pacientes mayores de sesenta años, con diagnóstico de dolor de cualquier etiología, que consultaron a la Fundación Santa Sofía para la Atención del Anciano, institución no gubernamental, dedicada a la atención de adultos mayores, ubicada en Bogotá D.C., en el período comprendido entre el primero de enero de 2001 y el 31 de diciembre de 2007.

La revisión de los registros clínicos, se efectuó con autorización de los directivos del centro y del Comité de Ética de la Institución, a través de la aplicación de un instrumento que incluyó los datos socio-demográficos de la población objeto de estudio, las características del dolor y el enfoque terapéutico utilizado.

Los criterios de inclusión de los registros clínicos fueron el diligenciamiento adecuado de los datos de la consulta, la legibilidad de la historia, la existencia de un diagnóstico de dolor de cualquier etiología, la claridad en el plan de manejo y la fecha de consulta en el lapso establecido, siendo descartadas las historias que no cumplieron todas las condiciones descritas anteriormente.

De 3956 historias clínicas identificadas en el período establecido, se llevó a cabo la eliminación de 2831 (71,57\%), como consecuencia del diligenciamiento incompleto de los datos mínimos requeridos para la inclusión en el estudio y/o de la falta de claridad en relación con las características de la consulta, quedando validados un total de 1125 registros clínicos, para su inclusión en el estudio.

Con la información adquirida, se elaboró una base de datos electrónica, que sirvió como insumo para el análisis descriptivo de los datos, en el programa Epi Info. El manejo de las historias clínicas y la recolección de la información cumplieron con los principios éticos universales y fueron evaluados y aprobados por el Comité de Ética de la Institución, instancia que recomendó tener en cuenta las cláusulas de confidencialidad establecidas universalmente, respecto a los datos de identificación de los pacientes y a la información de uso restringido, por parte del personal médico.

\section{RESULTADOS Y DISCUSIÓN}

En los datos obtenidos a partir de los registros clínicos $(n=1125)$, se apreció una mayor concentración de pacientes entre los 65 y los 74 años de edad (64\%), con una edad media de $71,5 \pm 5,5$ años y una clara predominancia del sexo femenino sobre el masculino, en una proporción de 4 a 1, siendo más notoria la diferencia en los grupos de mayores de 75 años de edad. Las características socio-demográficas básicas del grupo objeto de estudio pueden ser consultadas en la tabla 1.

Fueron clasificados 577 pacientes (51,3\%), en las categorías de dolor agudo y subagudo (entre un día y doce semanas de duración) y los 548 restantes (48,7\%), se ubicaron en la categoría de dolor crónico (más de doce semanas desde el inicio de la sintomatología). Las exacerbaciones agudas de cuadros con una base crónica fueron incluidas en el primer grupo, siendo contabilizados 268 casos (46,4\%) (Tabla 2).

De acuerdo con el mecanismo etiológico, la mayor proporción de registros fue incluida en la categoría dolor nociceptivo de origen somático $(73,6 \%)$, disponiéndose en segundo lugar la categoría de dolor nociceptivo de origen visceral $(13,8 \%)$ y, en el último, la categoría de dolor neuropático (12,6\%). No se reportaron casos de dolor de origen psicógeno, ni se evidenció la superposición de dos o más tipos de dolor (Tabla 2).

Según la localización, definida por sistemas orgánicos, el dolor de origen osteoarticular y/o muscular representó un $66,6 \%$, de todos los diagnósticos; el de origen gastrointestinal y genitourinario, un $13,9 \%$ y el originado en el sistema nervioso, un $13,2 \%$. El porcentaje restante correspondió a patologías generadas en otros sistemas y cuadros en que no se hizo explícito el mecanismo subyacente.

Tabla 1. Características socio-demográficas en pacientes observados.

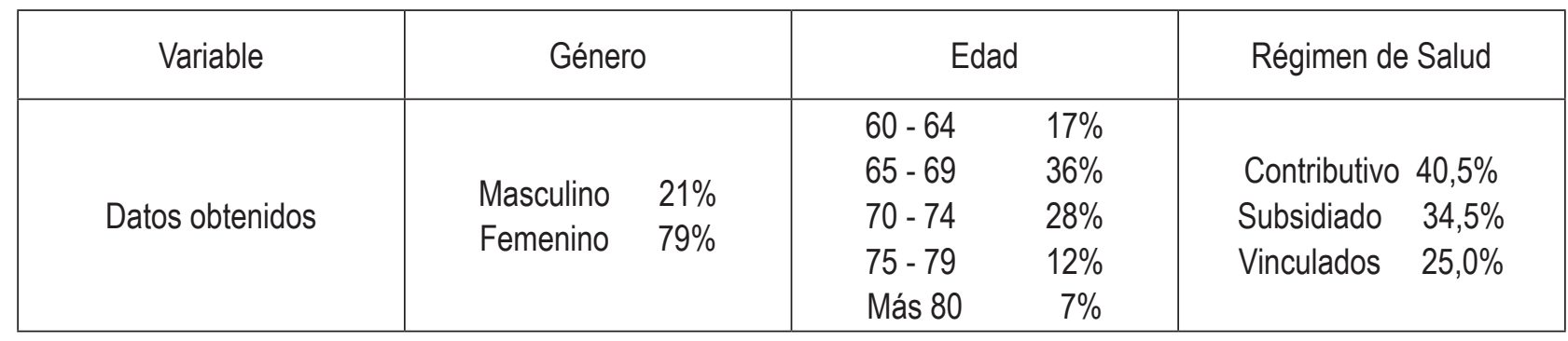


Tabla 2. Características clínicas del dolor.

\begin{tabular}{|c|c|c|c|c|c|c|c|}
\hline Aspecto & Patrón Temporal & Intensidad & Mecanismo Etiológico & & ación & & $\begin{array}{l}\text { ación } \\
\text { ional }\end{array}$ \\
\hline Resultados & $\begin{array}{ll}\text { Agudo } & 51,3 \% \\
\text { Crónico } & 48,7 \%\end{array}$ & $\begin{array}{lr}\text { Leve } & 35 \% \\
\text { Moderado } & 55 \% \\
\text { Severo } & 10 \%\end{array}$ & $\begin{array}{lc}\text { Somático } & 73,6 \% \\
\text { Visceral } & 13,8 \% \\
\text { Neuropático } & 12,6 \% \\
\text { Psicógeno } & --\end{array}$ & $\begin{array}{l}\text { Sí } \\
\text { No }\end{array}$ & $\begin{array}{l}18 \% \\
82 \%\end{array}$ & $\begin{array}{l}\text { Sí } \\
\text { No }\end{array}$ & $\begin{array}{l}70 \% \\
30 \%\end{array}$ \\
\hline
\end{tabular}

Únicamente en el $18 \%$ de las historias clínicas, se hizo mención de algún tipo de irradiación del dolor, siendo más frecuente los de origen neuropático. No obstante, en los registros clínicos restantes, no se evidenció, que confirme de forma explícita, la ausencia de irradiación a otros órganos o sistemas diferentes de los afectados, por lo que es imposible establecer la confiabilidad de los datos correspondientes, a esta categoría de análisis.

Respecto a la intensidad del dolor reportada en los registros clínicos analizados con base en los resultados de la aplicación de una escala numérica simple, se evidenció que la mayor proporción de pacientes experimentó dolor de intensidad moderada (55\%), seguido de dolor de intensidad leve (35\%) y severo (10\%) (Tabla 2$)$.

La presencia de algún grado de limitación funcional, se hizo patente en el $70 \%$ de los pacientes afectados; sin embargo, en la mayor parte de las historias clínicas revisadas, no se definieron con exactitud las características del fenómeno, ni se estableció con claridad el impacto sicológico y/o social del mismo (Tabla 2).

En cuanto al tipo de tratamiento, se utilizaron medidas no farmacológicas, en $7 \%$ de los casos; se instauró terapia farmacológica, en $42 \%$ de los pacientes; se empleó un esquema combinado, en $49 \%$ de los mismos y se aplicó otro tipo de intervención, en el porcentaje restante.

Para el tratamiento farmacológico, se aplicaron antiinflamatorios no esteroideos (38\%), acetaminofén (32\%), antiespasmódicos (13\%), relajantes musculares (6\%), asociaciones farmacológicas (AINEs y acetaminofén, relajantes musculares y acetaminofén, acetaminofén y tramadol), en $6 \%$ de los casos; otros agentes terapéuticos (antidepresivos tricíclicos, anticonvulsivantes, corticosteroides), en 3\% y opioides débiles (tramadol), en $2 \%$ de los casos.

La proporción de uso de antiinflamatorios no esteroideos inhibidores específicos de la COX 2, no se estableció con precisión, dado que en una gran parte de los registros clínicos analizados se incluyó la sigla genérica "AINEs" y no se explicitó el nombre genérico o comercial del compuesto manejado.
Como se mencionó anteriormente, los ancianos experimentan cuadros de dolor agudo y crónico con mayor frecuencia que otros grupos etáreos, apreciándose una gran atipicidad y complejidad en las quejas de estos pacientes; no obstante, existen relativamente pocos estudios orientados a evaluar el impacto del síntoma en la vida de ellos y las condiciones en que se llevan a cabo las acciones de diagnóstico y de tratamiento en estos pacientes (Gregorio Gil et al. 2007; Herr E Garand, 2001).

Este hecho no deja de sorprender, si se tiene en cuenta que la prevalencia estimada del dolor es dos veces más alta por encima de los sesenta años que en otros grupos de edad (250 por mil vs 125 por mil), encontrándose que una proporción cercana al $60 \%$ de los ancianos residentes en la comunidad y al $80 \%$ de los que se encuentran institucionalizados en hogares de larga estancia, experimentan algún tipo de dolor (Gregorio Gil et al. 2007; Herr \& Garand, 2001).

Por otra parte, la distribución de la población en relación con las variables demográficas analizadas en los registros clínicos, mayor prevalencia en el sexo femenino e incremento de la frecuencia del síntoma en relación directa con el aumento de la edad, concuerdan con los datos del Cuarto Estudio Nacional del Dolor (ACED, 2008) y con otros reportes (Gregorio Gil et al. 2007; Manson, 2010).

El nivel socio-económico no fue analizado por los investigadores como una variable que pudiera influenciar el comportamiento de los pacientes con dolor. En concordancia con lo anterior, los datos obtenidos en el Estudio Nacional del Dolor no sugieren la existencia de algún tipo de relación estadística, entre la presencia del síntoma y un estatus determinado (ACED, 2008).

La estimación de la frecuencia de dolor agudo respecto a la de dolor crónico está relacionada con la conceptualización empleada para su definición. Para tal efecto, en el presente estudio, se ha definido que la historia natural del dolor agudo no debe sobrepasar las 12 semanas, límite a partir del cual, se considera la existencia de cronicidad (Covarrubias-Gómez, 2006; Maestre, 2001; Hainline, 2005). 
Los datos asentados en esta investigación evidencian una frecuencia de dolor agudo, significativamente mayor que la reportada por algunos autores latinoamericanos (51,3\% versus $5 \%$ a $10 \%$ ), mientras que la de dolor crónico es inferior a la encontrada en estudios europeos (48,7\% versus $67,7 \%$ ), lo cual, puede ser explicado a partir del patrón temporal, utilizado como referencia y de la consideración de una tercera categoría intermedia, adoptada por algunos autores, denominada dolor subagudo, la que oscila entre una y doce semanas (Gregorio Gil et al. 2007; Covarrubias-Gómez, 2006; Maestre, 2001; Hainline, 2005).

Los tipos de dolor concuerdan con la distribución reportada en otros estudios llevados a cabo en este grupo poblacional y con la localización por sistemas, advirtiéndose una mayor frecuencia de dolor nociceptivo somático relacionado con patologías osteoarticulares y musculares, una proporción significativa de dolor nociceptivo de origen visceral (infección de vías urinarias, colitis de diversa etiología, gastroenteritis infecciosas y otras) y una proporción equivalente de dolor neuropático de diverso origen (Gregorio Gil et al. 2007; Covarrubias-Gómez, 2006; Hainline, 2005; Bruckental, 2008; Strassels et al. 2008).

Estos resultados concuerdan con el Reporte del Estudio Nacional del Dolor, aunque en este último, la localización del síntoma fue indagada a partir de un esquema topográfico, mientras que los datos obtenidos en este estudio fueron analizados desde un esquema de sistemas funcionales, siendo en cualquier caso, afectados con mayor frecuencia los segmentos corporales correspondientes al cráneo, la columna vertebral y sus anexos anatómicos, las extremidades y los sistemas gastrointestinal y genitourinario (ACED, 2008).

La referencia a la irradiación del dolor a otras áreas es baja y aunque no existen registros que permitan contrastar la información con otros estudios similares es importante resaltar que dicha característica clínica es más común en cuadros de dolor neuropático. La confiabilidad de los datos obtenidos en relación con la presencia o no de irradiación del dolor a otros segmentos corporales es baja, dada la imposibilidad de confirmar la existencia del fenómeno en las recolecciones clínicas. En términos generales, los datos conseguidos a partir de la revisión de los registros clínicos en relación con la variable intensidad del dolor, coinciden con los reportados en el Estudio Nacional del Dolor y otros estudios relacionados, percibiéndose algunas limitaciones en los instrumentos utilizados (Dijkers, 2010; Gregorio Gil et al. 2007; ACED, 2008; Feldt, 2007).

La intensidad es una de las variables de mayor relevancia durante el abordaje diagnóstico y terapéutico del paciente con dolor; sin embargo, existen otras dimensiones que deben ser exploradas durante la evaluación del paciente con dolor, por lo que se hace necesario considerar la aplicación de escalas multidimensionales que permitan una mejor aproximación a la comprensión del fenómeno nociceptivo, desde las perspectivas biológica, sicológica y social (Dijkers, 2010; Gregorio Gil et al. 2007; Feldt, 2007)

Para tal efecto, el Consenso Interdisciplinario para la Evaluación del Dolor en Adultos Mayores, recomienda la aplicación del BPI (Brief Pain Inventory) y del SF MPQ (Short Form McGill Pain Questionnaire), como herramientas, que permiten una valoración adecuada del síntoma, desde un enfoque que garantice la integración de las variables que condicionan la naturaleza del mismo (Feldt, 2007; Hadjistavropoulus et al. 2007; Deane E Smith, 2008).

El 70\% de los registros clínicos analizados incluyeron alguna referencia a la existencia de limitación funcional; no obstante, solamente una proporción mínima de las historias contenía datos explícitos acerca del grado de disfuncionalidad, de la naturaleza de dicha alteración y del impacto del fenómeno en la autonomía del paciente, siendo necesario prestar mayor atención a este hecho, con el fin de mejorar la calidad de vida de los pacientes ancianos con dolor (Leonhardt $\mathcal{E}$ Laekeman, 2010).

Como una referencia indirecta al fenómeno, vale la pena mencionar que una proporción elevada de los americanos mayores de 65 años presentaba algún tipo de discapacidad relacionada con dolor, en un estudio llevado a cabo en el 2000; esta proporción, se pudo haber incrementado en los últimos años, como consecuencia del crecimiento acelerado de este grupo poblacional (Deane E Smith, 2008).

La intensificación de los síntomas en las últimas horas del día, se hizo evidente en dos terceras partes de los registros clínicos analizados; este hecho podría ser explicado, a partir de la organización de los ciclos circadianos de algunos sistemas del organismo humano; sin embargo, en la revisión bibliográfica, no se hallaron referencias específicas respecto a esta característica del dolor.

La persistencia del síntoma en un $25 \%$ de los casos es consistente con datos disponibles en la literatura, reportándose, en algunos estudios, una proporción dos o tres veces más alta, la cual, podría estar relacionada con los parámetros utilizados para la definición del concepto referido (Maxwell et al. 2008).

La aplicación de medidas no farmacológicas en forma aislada (ejercicio físico, pérdida de peso, uso de dispositivos de asistencia, educación) es una buena opción terapéutica en procesos de dolor agudo de intensidad leve; no obstante, 
en este estudio, sólo fue contemplada en una proporción mínima de los casos analizados. En la mitad de los pacientes, se implantó un esquema combinado, que incluyó terapias alternativas y medicamentos para el control del dolor, lo que ha sido reportado en la literatura, como un esquema válido para el manejo del dolor, de origen músculo esquelético (Am. Soc. Anesthesiol. Task Force on Chronic Pain Management, 2010; Weiner, 2007).

Diferentes autores recomiendan que el tratamiento del dolor en el anciano se enfoque siguiendo la escalera analgésica de la Organización Mundial de la Salud, aunque es necesario advertir, al respecto, que dichos individuos tienen una mayor predisposición a presentar efectos adversos de diferente naturaleza. Por otra parte, debido a la gran variabilidad individual de estos individuos, no es fácil establecer protocolos genéricos, razón por la que vale la pena acotar el concepto de la Sociedad Americana de Geriatría referente al tratamiento farmacológico de los adultos mayores de "empezar lento y continuar despacio", para valorar, de forma adecuada, la respuesta al tratamiento y los efectos secundarios del mismo (Gregorio Gil et al. 2007; Franco E Lucas, 2001).

La frecuencia de utilización de AINES, en este estudio, coincide con informes de otros autores; en contraste, se presentan diferencias significativas respecto al uso de acetaminofén. De acuerdo con los esquemas actuales, los AINEs son la primera opción terapéutica en dolor nociceptivo de origen somático; pero vale la pena recordar la gran frecuencia de efectos adversos en los sistemas gastrointestinal, cardiovascular y renal, en este grupo de edad (Loza, 2008; Gregorio Gil et al. 2007).

En el estudio, no se identificaron razones válidas para explicar la baja frecuencia del uso de acetaminofén, especialmente, si se tiene en cuenta que este agente se encuentra indicado como terapia de primera línea en dolor leve a moderado, en ausencia de inflamación, marcada por el bajo riesgo de eventos adversos asociados (Gregorio Gil et al. 2007; Franco E Lucas, 2001; Horgas, 2003).

El uso de opiáceos está incluido en el segundo y en el tercer escalón de la escalera analgésica de la OMS, para el tratamiento del dolor moderado a severo; sin embargo, en nuestro estudio, no ocupó un lugar preponderante, probablemente, como consecuencia de la reticencia que produce el uso de dichos medicamentos en dolor no maligno, dada la frecuencia de efectos adversos y la probabilidad de adicción en los ancianos, hechos que no justifican la falta de control adecuado del dolor, en los casos en que se encuentren indicados (Gregorio Gil et al. 2007; Franco E Lucas, 2001; Ford, 1999).

Por otra parte, las asociaciones farmacológicas representan una buena opción en aquellos casos en que el tratamiento de un solo medicamento no permite el control total del síntoma. De acuerdo con el esquema de manejo planteado en la escalera analgésica, el uso de opiáceos débiles en combinación con no opiáceos está indicado en dolor nociceptivo de origen somático $\mathrm{y}$, en algunos casos, de dolor neuropático de intensidad moderada a severa (Woolf \& Mannion, 1999; Ford, 1999; Gómez-Cortés E Rodríguez-Huertas, 2000).

Vale la pena anotar que la revisión de los registros clínicos que cumplieron con los criterios de inclusión fue complementada con un análisis del acto médico, correspondiente a la luz del enfoque bio-sicosocial de la salud, verificándose que solamente en una proporción mínima de los registros (1,87\%) existían indicios de que la consulta hubiese estado enmarcada en un modelo de atención integral, que incluyera la evaluación de las características biológicas, sicológicas, afectivas, funcionales y sociales del dolor, así como la influencia del contexto y el ambiente en la génesis y evolución del cuadro.

A pesar de las limitaciones que surgen a partir del planteamiento de una visión retrospectiva de un fenómeno clínico tan complejo como el dolor y de las dificultades para estandarizar el registro del mismo en las historias clínicas utilizadas como fuente de información, el trabajo permite establecer una caracterización básica de los elementos clínicos, que configuran la experiencia nociceptiva en el adulto mayor y aporta algunos elementos de reflexión, en torno al diagnóstico $\mathrm{y}$ al tratamiento de estos pacientes.

La baja proporción de historias clínicas que reflejaron el enfoque del cuadro a partir de un modelo bio-sicosocial de la enfermedad es un elemento que genera grandes interrogantes acerca del marco conceptual, dispuesto como referencia de la práctica médica, en contraste con lo expresado en la literatura, respecto a la necesidad de considerar los aspectos biológico, sicológico, social y cultural, como ejes centrales del abordaje diagnóstico y terapéutico de cualquier patología (Am. Soc. Anesthesiol. Task Force on Chronic Pain Management, 2010).

Así, el manejo adecuado del paciente con dolor requiere la implantación de un modelo integrado de abordaje biosicosocial que debe estar fundamentado en el trabajo de un equipo interdisciplinario responsable de la valoración detallada de los siguientes aspectos (Am. Soc. Anesthesiol. Task Force on Chronic Pain Management, 2010; Gregorio Gil et al. 2007):

- Historia médica completa, haciendo énfasis en las características del dolor y examen clínico detallado, centrado en los síntomas expresados por el paciente y en los sistemas óseo, articular, muscular y neurológico, en los casos que lo ameriten. 
- Historia sicológica integral, incluyendo diagnóstico del perfil sicológico, historia del paciente en relación con el dolor (experiencia personal, aspectos cognitivos y afectivos), repercusiones conductuales y estrategias de afrontamiento.

- Historia social, centrada en la influencia de lo social, en la génesis del dolor y en la repercusión de este último sobre las relaciones familiares y sociales del paciente (antecedentes familiares, nivel de actividad, respuesta a estrés del medio, historia conyugal, entre otros).

- Historia funcional, tendiente a determinar el impacto del dolor en la autonomía del paciente y las repercusiones clínicas, mentales y sociales del mismo en la vida cotidiana.

- Aplicación de escalas de medición del dolor. El uso de instrumentos especializados de medición permite objetivizar las características del dolor y establecer el impacto real del mismo en las esferas clínica, funcional, mental y social. Los dos instrumentos recomendados para uso en la consulta de atención primaria son el Brief Pain Inventory (BPI) y la versión corta del McGill Pain Questionnaire.

Consecuente con lo anterior, un modelo adecuado de intervención del paciente anciano con dolor crónico, a partir de un enfoque bio-sicosocial, debe estar fundamentado en la planeación e implementación de estrategias orientadas a la rehabilitación integral del paciente, incluyendo:

- Farmacoterapia específica, idealmente orientada según el mecanismo etiológico del dolor.

- Procedimientos coadyuvantes especializados (acupuntura, terapia neural, anestesia local o regional).

- Utilización de técnicas psicoterapéuticas de enfoque conductual, cognitivo conductual, psicodinámico y/o sistémico.

- Educación en técnicas de manejo de estrés, entrenamiento de asertividad, técnicas de relajación y autohipnosis.

- Terapias de rehabilitación y de promoción de la reinserción a la vida cotidiana (fisioterapia, terapia ocupacional, integración a grupos de pares).

- Remisión especializada a los diferentes miembros del equipo interdisciplinario para el manejo del dolor.

Agradecimientos. A la Universidad de Ciencias Aplicadas y Ambientales U.D.C.A por el apoyo financiero para la realización de este estudio. Conflictos de interés: El manuscrito fue preparado y revisado con la participación de todos los autores, quienes declaramos que no existe ningún conflicto de intereses que ponga en riesgo la validez de los resultados presentados.

\section{BIBLIOGRAFÍA}

1. AMERICAN SOCIETY OF ANESTHESIOLOGISTS TASK FORCE ON CHRONIC PAIN MANAGEMENT. 2010. Practice guidelines for chronic pain management. Anesthesiology. 112: 810-833.

2. ASHBURN, M.; STAATS, P. 1999. Management of chronic pain. Lancet. 353:1865-1869.

3. ASOCIACIÓN COLOMBIANA PARA EL ESTUDIO DEL DOLOR, ACED. 2008. Estudio Nacional del Dolor. Disponible desde Internet en: http://www.dolor.org.co/ index.php?option $=$ com_contentEview $=$ articleEid $=6$ $8 \mathcal{E}$ Itemid=88 (con acceso 13/08/09).

4. BORSOOK, D.; Le BEL, A.; Mc PEEK, B. 1999. The Massachusetts General Hospital Handbook of pain management. Ed. Marbán (U.S.A). p.85-110, 134-138.

5. BRUCKENTAL, P. 2008. Assessment of pain in the elderly adult. Clin. Geriatr. Med. 4:213-236.

6. CATANANTI, C.; GAMBASSI, G. 2010. Pain assessment in the elderly. Surgical Oncology. 19(3):140-148.

7. CHRISTO, P. 2003. Opioid effectiveness and side effects in chronic pain. Anesthesiol. Clin. North America. 21(4):699-713.

8. CLARK, M.; COX, T. 2002. Refractory Chronic Pain. Psychiatric Clinics North America. 25:71-88.

9. COVARRUBIAS-GÓMEZ, A. 2006. Manejo del dolor en el paciente anciano. Rev. Mex. Anestesiología. 29(S1):207209.

10. DEANE, G.; SMITH, H. 2008. Overview of pain management in older Persons. Clin Geriatr Med. 1:85-201.

11. DIJKERS, J. 2010. Comparing quantification of pain severity by verbal rating and numeric scales. J. Spinal Cord Medicine. 33(3):232-242.

12. FELDT, K. 2007. Pain measurement: present concerns and future directions. Pain Medicine. 8(7):1-4.

13. FRANCO, M.L.; de LUCAS, S. 2001. Características del dolor crónico en el anciano: tratamiento. Rev. Soc. Esp. Dolor. 8:29-38. 
14. FORD, E. 1999. The challenge of pain management in the elderly patient in Hospice Care. J. Hospice and Palliative Nursing. 1(1):9-18.

15. GÓMEZ-CORTÉS, M.; RODRÍGUEZ-HUERTAS, F. 2000. Revaluación del segundo escalón de la escalera analgésica de la OMS. Rev Soc. Esp. Dolor. 7:343-344.

16. GOTTSCHALK, A.; SMITH, D. 2001. New concepts in acute pain therapy: preemptive analgesia. Am. Fam. Physician. 63:1979-1984, 1985-1986.

17. GREGORIO, P.; MORENO, A.; RODRÍGUEZ, M.J.; ZARCO, J. 2007. Manejo del dolor del anciano en atención primaria (ADA). Rev. Clin. Esp. 207(4):166-171.

18. HADJISTAVROPOULUS, T.; HERR, K.; TURK, D.; FINE, D.; DWORKIN, R.; HELME, R.; JACKSON, K.; PHARM, D.; PARMELEE, P.; RUDY, T.; BEATTIE, L.; CHIBNALL, J.; CRAIG, K.; FERRELL, B.; FILLINGIM, R.; GAGLIESE, L.; GALLAGHER, R.; GIBSON, S.; HARRISON, E.; KATZ, B.; KEEFE, F.; LIEBER, S.; LUSSIER, D.; SCHMADER, K.; TAIT, R.; WEINER, D.; WILLIAMS, J. 2007. An interdisciplinary expert consensus statement on assessment of pain in older persons. Clin. J. Pain. 23(1):S1-S43.

19. HAINLINE, B. 2005. Chronic pain: physiological, diagnostic and management considerations. Psych. Clin. North America. 28(3):723-735.

20. HERR, K.; GARAND, L. 2001. Pain management in the elderly: assessment and measurement of pain in older adults. Clin. Ger. Med. 17(3):1-26.

21. HORGAS, A. 2003. Pain management in elderly adults. J. Infusion Nursing. 26(3):161-165.

22. KATZ, J. 1999. Measurement of pain. Pain control in the perioperative period. Surg. Clin. North America. 79(2):115-123.
23. LEONHARDT, C.; LAEKEMAN, M. 2010. Pain and fear of movement in the elderly: The need for an interdisciplinary approach. Der Schmerz (Alemania). Abstract, pre-publicación.

24. LOZA, E. 2008. Revisión sistemática: ces eficaz y seguro el uso de AINE para los ancianos? Reumatol Clin. 4(5):172-182.

25. MAESTRE, J.A. 2001. El dolor en el adulto mayor. Colombia Médica. 32(4):184-188.

26. MANSON, J.E. 2010. Pain: Sex differences and implications for treatment. Metabolism: clinical and experimental. 59(Suppl 15):S16-20.

27. MAXWELL, C.; DALBY, D.; SLATER, M.; PATTEN, S.; HORGAN, D.; ELIASZIW, M.; HIRDES, J. 2008. The prevalence and management of current daily pain among older home care clients. Pain. 1:208-216.

28. MARCUS, D. 2000. Treatment of nonmalignant chronic pain. Am. Family Physician. 61:1331-1338, 1345-1346.

29. STRASSELS, S.; Mc NICOL, E.; SULEMAN, R. 2008. Pharmacotherapy of pain in older adults. Clin. Geriatr. Med. 24:275-298.

30. TURK, D.; OKIFUJI, A. 2001. Assessment of patients reporting of pain: An integrated perspective. Lancet. 357: Suppl 1:S1-S7.

31. WEINER, D. 2007. Office management of chronic pain in the elderly. Am. J. Med. 120:306-315.

32. WOOLF, C.; MANNION, R. 1999. Neuropathic pain: aetiology, symptoms, mechanisms and management. Lancet. 353:1959-1964.

Recibido: Febrero 24 de 2010

Aceptado: Octubre 26 de 2010 